\title{
AN ENTROPIC UNCERTAINTY PRINCIPLE FOR POSITIVE OPERATOR VALUED MEASURES
}

\author{
MICHEL RUMIN
}

\begin{abstract}
Extending a recent result by Frank and Lieb, we show an entropic uncertainty principle for mixed states in a Hilbert space, relatively to pairs of positive operator valued measures that are independent in some sense. This yields spatial-spectral uncertainty principles and log-Sobolev inequalities for invariant operators on homogeneous spaces, which are sharp in the compact case.
\end{abstract}

\section{INTRODUCTION AND MAIN RESULT}

A mixed state (or density matrix) in a Hilbert space $H$ is a positive operator $\rho$ on $H$ with trace $\tau(\rho)=1$. From the quantum view-point, one can measure $\rho$ through positive operator valued measures (POVM) on $H$. We briefly recall this.

A POVM is a countably additive map $P$ from a $\sigma$-algebra on a space $X$ into the positive operators on $H$ and such that $P(X)=1_{H}$, see [11, 18]. Important examples are given by the following cases:

- When $H$ is isometric to a function space $L^{2}(X, \mu)$, one can set $P(\Omega)$ to be the multiplication by the characteristic function $\chi_{\Omega}$ for measurable sets $\Omega \subset X$. This is actually a projection valued measure.

- Other projection valued measures come from the spectral resolution of a self-adjoint operator $A$ on $H$. Here $X=\mathbb{R}$ and $P(I)=\Pi_{A}(I)$ is the spectral projector of $A$ associated to $I \subset \mathbb{R}$.

- Partition of unity in $H$, i.e. series of positive operators $P_{i}$ such that $\sum_{i} P_{i}=1_{H}$.

A state $\rho$ and a POVM induce a probability measure $\nu_{P}^{\rho}$ on $X$ by

$$
\nu_{P}^{\rho}(\Omega)=\tau(\rho P(\Omega))=\tau\left(\rho^{1 / 2} P(\Omega) \rho^{1 / 2}\right),
$$

that encodes the result of the measurement of $\rho$ through $P$.

The statement we want to discuss deals with the measures $\nu_{P}^{\rho}$ and $\nu_{Q}^{\rho}$ associated to a pair of POVM on $H$, say $P$ from $X$ and $Q$ from $Y$. This pair induces a state-independent measure $\mu_{P Q}$ on $X \times Y$, called a 'Liouville measure' from now on, and defined by

$$
\mu_{P Q}\left(\Omega_{1} \times \Omega_{2}\right)=\tau\left(P\left(\Omega_{1}\right) Q\left(\Omega_{2}\right)\right)=\tau\left(P\left(\Omega_{1}\right)^{1 / 2} Q\left(\Omega_{2}\right) P\left(\Omega_{1}\right)^{1 / 2}\right) .
$$

Date: October 31, 2018.

2000 Mathematics Subject Classification. 58J42, 58J50, 47B06, 43A85, 94A17.

Key words and phrases. entropy, POVM, uncertainty principle, homogeneous spaces, log-Sobolev. 
We shall see that when the Liouville measure $\mu_{P Q}$ is bounded by a $\sigma$-finite product measure $\mu_{P} \otimes \mu_{Q}$, then $\nu_{P}^{\rho} \ll \mu_{P}$ and $\nu_{Q}^{\rho} \ll \mu_{Q}$. Let then

$$
\varrho_{P}=\frac{d \nu_{P}^{\rho}}{d \mu_{P}} \quad \text { and } \quad \varrho_{Q}=\frac{d \nu_{Q}^{\rho}}{d \mu_{Q}}
$$

denote the corresponding density functions. Our main result is the following.

Theorem 1.1. Using the notations above, suppose that $\mu_{P Q} \leq \mu_{P} \otimes \mu_{Q}$ for some $\sigma$-finite product measure on $X \times Y$. Then it holds

$$
-\int_{X} \varrho_{P} \ln \varrho_{P} d \mu_{P}-\int_{Y} \varrho_{Q} \ln \varrho_{Q} d \mu_{Q} \geq S(\rho)=-\tau(\rho \ln \rho),
$$

provided the left side is well defined, i.e. we don't face $\infty-\infty$ in the integrals and their sum.

Here $S(\rho)$ is the intrinsic von Neumann entropy of the state, independent on any choice of POVM, while the integrals are minus the relative entropy from $\nu_{P}^{\rho}$ to $\mu_{P}$ and $\nu_{Q}^{\rho}$ to $\mu_{Q}$. Hence (1) is an entropic uncertainty principle claiming that the $P$ and $Q$-measurements of $\rho$ can't be both concentrated in small sets for $\mu_{P}$ and $\mu_{Q}$, under some hypothesis on the Liouville measure. From the probabilistic view-point, the assumption on $\mu_{P Q}$ deals with the independence of the two POVM. The best case occurs when $\mu_{P Q}$ is a product measure itself: and we shall say that $P$ are $Q$ are independent there. We give some examples.

\section{First exAmples}

2.1. Pair of bases. Let $\left(e_{i}\right)$ and $\left(f_{i}\right)$ be two orthonormal bases of a Hilbert space $H$. This gives two projector valued measures from (finite or not) sets $X=Y \subset \mathbb{Z}$ with $P(i)=\Pi_{e_{i}}$ and $Q(i)=\Pi_{f_{i}}$. The measurements of $\rho$ are

$$
\nu_{P}^{\rho}(i)=\left\langle\rho e_{i}, e_{i}\right\rangle=p_{i}, \quad \nu_{Q}^{\rho}(j)=\left\langle\rho f_{j}, f_{j}\right\rangle=q_{j}
$$

and the Liouville measure is

$$
\mu_{P Q}(i, j)=\tau(P(i) Q(j))=\left|\left\langle e_{i}, f_{j}\right\rangle\right|^{2} .
$$

Clearly, it holds that $\mu_{P Q} \leq \mu_{P} \otimes \mu_{Q}$ with

$$
\mu_{P}(i)=\sup _{j}\left|\left\langle e_{i}, f_{j}\right\rangle\right| \text { and } \mu_{Q}(j)=\sup _{i}\left|\left\langle e_{i}, f_{j}\right\rangle\right| .
$$

Theorem 1.1 yields

$$
\begin{gathered}
-\sum_{i} p_{i} \ln p_{i}-\sum_{j} q_{j} \ln q_{j} \geq S(\rho)+K, \\
\text { with } \quad K=-\sum_{i} p_{i} \ln \mu_{P}(i)-\sum_{j} q_{j} \ln \mu_{Q}(j) \geq 0 .
\end{gathered}
$$

This improves a result by Frank and Lieb in [7] where $K$ is replaced by

$$
K^{\prime}=-2 \ln \left(\sup _{i, j}\left|\left\langle e_{i}, f_{j}\right\rangle\right|\right) \leq K .
$$


The better estimation is obtained when $P$ and $Q$ are independent, i.e. when $\mu_{P Q}$ is a product measure. Taking partial $i$ and $j$ sums, one sees that this only happens in finite dimension $d$ and when

$$
\left|\left\langle e_{i}, f_{j}\right\rangle\right|^{2}=d^{-1}
$$

which is a definition of mutually unbiased bases, used in quantum information theory [11, 17.

We note that such results generalize to pairs of partitions of unity $P_{i}$ and $Q_{j}$ obtained in the following way. Given two orthonormal bases $\left(e_{i}\right)$ and $f_{j}$ of Hilbert space $H^{\prime}$ containing $H$, one gets two POVM on $H$ by setting

$$
P_{i}=\Pi_{H} \Pi_{e_{i}} \Pi_{H} \quad \text { and } \quad Q_{j}=\Pi_{H} \Pi_{f_{j}} \Pi_{H} .
$$

One finds that the same inequality as (2) holds replacing $e_{i}$ and $f_{j}$ by $\Pi_{H} e_{i}$ and $\Pi_{H} f_{j}$ in formulas.

2.2. Fourier transform and uncertainty. Consider now $H=L^{2}\left(\mathbb{R}^{n}, d x\right)$ with its natural projection valued measure from $X=\mathbb{R}_{x}^{n}$ defined by $P(\Omega)=\chi_{\Omega} \times$. Fourier transform on $H$, with convention that

$$
\mathcal{F}(f)(\xi)=\int_{\mathbb{R}^{n}} f(x) e^{-2 i \pi\langle x, \xi\rangle} d x
$$

is an isometry onto $L^{2}\left(\mathbb{R}_{\xi}^{n}, d \xi\right)$, and provides a second projection valued measure $Q$ from $Y=\mathbb{R}_{\xi}^{n}$ into $H$ by setting $Q(\Omega)=\mathcal{F}^{-1} \chi_{\Omega} \mathcal{F}$.

These two maps are independent in our sense. Indeed, our 'Liouville measure' is here

$$
\begin{aligned}
\mu_{P Q}\left(\Omega_{1} \times \Omega_{2}\right) & =\tau\left(\chi_{\Omega_{1}} \mathcal{F}^{*} \chi_{\Omega_{2}} \mathcal{F}\right)=\left\|\chi_{\Omega_{2}} \mathcal{F} \chi_{\Omega_{1}}\right\|_{H S}^{2} \text { (Hilbert Schmidt norm) } \\
& =\int_{\Omega_{2} \times \Omega_{1}}\left|e^{-2 i \pi\langle x, \xi\rangle}\right|^{2} d \xi d x \quad \text { (using kernels) } \\
& =\lambda\left(\Omega_{1}\right) \lambda\left(\Omega_{2}\right)
\end{aligned}
$$

i.e. the (genuine) Liouville measure of the phase space $\mathbb{R}_{x}^{n} \times \mathbb{R}_{\xi}^{n}$.

We make explicit Theorem 1.1. A state may be written $\rho=\sum_{i} p_{i} \Pi_{f_{i}}$ for orthonormal functions $f_{i}$. Then its measurement in $P$ reads

$$
\nu_{P}^{\rho}(\Omega)=\tau\left(\rho \chi_{\Omega}\right)=\int_{\Omega} \sum_{i} p_{i}\left|f_{i}(x)\right|^{2} d x,
$$

while from $Q$

$$
\begin{aligned}
\nu_{Q}^{\rho}(\Omega) & =\tau\left(\rho \mathcal{F}^{-1} \chi_{\Omega} \mathcal{F}\right)=\tau\left(\mathcal{F} \rho \mathcal{F}^{-1} \chi_{\Omega}\right) \\
& =\int_{\Omega} \sum_{i} p_{i}\left|\mathcal{F}\left(f_{i}\right)(\xi)\right|^{2} d \xi,
\end{aligned}
$$

since $\mathcal{F} \rho \mathcal{F}^{-1}=\sum_{i} p_{i} \Pi_{\mathcal{F}\left(f_{i}\right)}$. Finally, Theorem 1.1 states that

$$
-\int_{\mathbb{R}^{n}} \varrho(x) \ln \varrho(x) d x-\int_{\mathbb{R}^{n}} \widehat{\varrho}(\xi) \ln \varrho(\xi) d \xi \geq S(\rho)=-\sum_{i} p_{i} \ln p_{i}
$$

with

$$
\varrho(x)=\frac{d \nu_{P}^{\rho}}{d x}=\sum_{i} p_{i}\left|f_{i}(x)\right|^{2} \quad \text { and } \quad \widehat{\varrho}(\xi)=\frac{d \nu_{Q}^{\rho}}{d \xi}=\sum_{i} p_{i}\left|\mathcal{F}\left(f_{i}\right)(\xi)\right|^{2}
$$


This inequality has been first proved by Frank and Lieb in [7], and is known to be sharp for states $\rho_{t}=e^{-t\left(\Delta+\|x\|^{2}\right)} / \tau\left(e^{-t\left(\Delta+\|x\|^{2}\right)}\right)$ when $t \searrow 0$.

2.3. Frank and Lieb result. The previous example is actually a particular case of a more general theorem proved in [7].

Suppose that two $\sigma$-finite function spaces $L^{2}(X, \mu)$ and $L^{2}(Y, \nu)$ are isometric through

$$
\mathcal{U}: L^{2}(X, \mu) \rightarrow L^{2}(Y, \nu) .
$$

Suppose moreover that $\mathcal{U}$ is bounded from $L^{1}(X, \mu)$ to $L^{\infty}(Y, \nu)$. Let $\rho=\sum_{i} p_{i} \Pi_{f_{i}}$ be a (unit trace) state on $L^{2}(X, \mu)$ and consider $\widehat{\rho}=\mathcal{U} \rho \mathcal{U}^{-1}=\sum_{i} p_{i} \Pi_{\mathcal{U} f_{i}}$ on $L^{2}(Y, \nu)$. Let

$$
\varrho(x)=\sum_{i} p_{i}\left|f_{i}(x)\right|^{2} \quad \text { and } \quad \widehat{\varrho}(\xi)=\sum_{i} p_{i}\left|\mathcal{U}\left(f_{i}\right)(\xi)\right|^{2}
$$

be their density as above.

Theorem 2.1. [7, Thm 2.2] Suppose moreover that

$$
\int_{X} \varrho(x) \ln ^{+} \varrho(x) d \mu(x)<+\infty \quad \text { and } \quad \int_{Y} \widehat{\varrho}(y) \ln ^{+} \widehat{\varrho}(y) d \nu(y)<+\infty
$$

Then

$$
-\int_{X} \varrho(x) \ln \varrho(x) d \mu(x)-\int_{Y} \widehat{\varrho}(y) \ln \widehat{\varrho}(y) d \nu(y) \geq S(\rho)-2 \ln \|\mathcal{U}\|_{L^{1} \rightarrow L^{\infty}} .
$$

Theorem 1.1 implies it. Indeed, as in the previous discussion on Fourier transform, one defines two projections valued measures on $H=L^{2}(X, \mu)$ : the standard one $P(\Omega)=\chi_{\Omega} \times$ from $X$, and $Q(\Omega)=\mathcal{U}_{\chi} \mathcal{U}^{-1}$ from $Y$. Then arguing as previously, one has

$$
\begin{aligned}
\mu_{P Q}\left(\Omega_{1} \times \Omega_{2}\right) & =\tau\left(\chi_{\Omega_{1}} \mathcal{U} \chi_{\Omega_{2}} \mathcal{U}^{*}\right)=\left\|\chi_{\Omega_{2}} \mathcal{U}^{*} \chi_{\Omega_{1}}\right\|_{H S}^{2} \\
& =\int_{\Omega_{1} \times \Omega_{2}}|\mathcal{U}(y, x)|^{2} d \mu(x) d \nu(y) \\
& \leq \mu\left(\Omega_{1}\right) \nu\left(\Omega_{2}\right)\|\mathcal{U}\|_{L^{1} \rightarrow L^{\infty}}^{2} .
\end{aligned}
$$

This shows that Theorem 1.1 applies and yields (4).

Comparing to Theorem 2.1. Theorem 1.1 adds some flexibility in the choice of the mesures on $X$ and $Y$, and applies to general positive operator valued measures, as illustrated in $\$ 2.1$. Here the mesures on $X$ and $Y$ are not fixed a priori but adapted to the interaction of the two maps $P$ and $Q$ through the intrisic Liouville measure. We note also that Theorem 2.1 deals with $L^{2}$ function spaces, while Theorem 1.1 applies to general direct integral decompositions of Hilbert spaces, as for instance to splittings $H=\oplus_{\lambda} E_{\lambda}$ with non constant $\operatorname{dim} E_{\lambda}$ coming from spectral resolution of operators. See examples in $\S$.

Another feature of Theorem 1.1 is its invariance through general isometries of $H$. Indeed, if an isometry $U$ acts on $H$, then two initial POVM $P$ and $Q$ are conjugated to $P^{U}=U^{-1} P U$ and $Q^{U}=U^{-1} Q U$. The Liouville measure is preserved: $\mu_{P^{U} Q^{U}}=\tau\left(P^{U} Q^{U}\right)=\tau(P Q)=\mu_{P Q}$, as the measures associated to $\rho$ and $\rho^{U}: \nu_{P}^{\rho}=\nu_{P U}^{\rho^{U}}$ and $\nu_{Q}^{\rho}=\nu_{Q^{U}}^{\rho^{U}}$. In comparison, (ब) is not invariant since the underlying isometry $\mathcal{U}_{F L}$ there becomes $U^{-1} \mathcal{U}_{F L} U$, whose $L^{1} \rightarrow L^{\infty}$ norm is a priori not controlled by $\left\|\mathcal{U}_{F L}\right\|_{1, \infty}$ alone. 


\section{Proof of Theorem 1.1 And Related Comments}

Our proof of Theorem 1.1 follows the lines of Frank and Lieb's argument for Theorem 2.1. It relies on two classical lemmas in quantum statistical mechanics, see e.g. [2] and [15, Thm. 8.5].

Lemma 3.1 (Gibbs variational formula). Let $A$ be a self-adjoint operator such that $e^{-A}$ is trace class. Then for any unit trace state $\rho$, it holds

$$
\tau(A \rho)-S(\rho) \geq-\ln \tau\left(e^{-A}\right),
$$

with equality iff $\rho=e^{-A} / \tau\left(e^{-A}\right)$.

Lemma 3.2 (Golden-Thompson inequality). Let $A$ and $B$ be self-adjoint operators with upper bounds. Then it holds that

$$
\tau\left(e^{A+B}\right) \leq \tau\left(e^{A / 2} e^{B} e^{A / 2}\right) .
$$

Here, following [9], $A+B$ stands for the self-adjoint operator associated to the closed upperbounded quadratic form $q_{A}+q_{B}$ on the closure of $D\left(A_{-}^{1 / 2}\right) \cap D\left(B_{-}^{1 / 2}\right)$.

We suppose that $\mu_{P Q} \leq \mu_{P} \otimes \mu_{Q}$ and first check the absolute continuity of $\nu_{P}^{\rho}$ and $\nu_{Q}^{\rho}$ with respect to $\mu_{P}$ and $\mu_{Q}$. If $\mu_{P}(\Omega)=0$, then by $\sigma$-finiteness of $\mu_{Q}$ one has $\mu_{P Q}(\Omega \times Y)=$ $\tau(P(\Omega))=0$. Hence $P(\Omega)=0$, and $\nu_{P}^{\rho}(\Omega)=\tau(\rho P(\Omega))=0$ as claimed.

Given $k \geq 0$, let $\varrho_{P, k}=\min \left(\varrho_{P}, k\right)$ and $\varrho_{Q, k}=\min \left(\varrho_{Q}, k\right)$. We consider the following bounded positive operators on $H$

$$
A_{P}=\int_{X} \varrho_{P, k} d P \quad \text { and } \quad A_{Q}=\int_{Y} \varrho_{Q, k} d Q .
$$

Using a Hilbert basis $\left(e_{i}\right)$ of $H$ and monotone convergence, one sees that

$$
\tau\left(A_{P}^{1 / 2} A_{Q} A_{P}^{1 / 2}\right)=\sum_{i}\left\langle A_{Q} A_{P}^{1 / 2} e_{i}, A_{P}^{1 / 2} e_{i}\right\rangle=\int_{Y} \varrho_{Q, k} \tau\left(A_{P}^{1 / 2} d Q A_{P}^{1 / 2}\right)
$$

where for any measurable set $\Omega^{\prime} \subset Y$

$$
\begin{aligned}
\tau\left(A_{P}^{1 / 2} Q\left(\Omega^{\prime}\right) A_{P}^{1 / 2}\right) & =\tau\left(Q^{1 / 2}\left(\Omega^{\prime}\right) A_{P} Q^{1 / 2}\left(\Omega^{\prime}\right)\right) \\
& =\int_{X} \varrho_{P, k} \tau\left(Q^{1 / 2}\left(\Omega^{\prime}\right) d P Q^{1 / 2}\left(\Omega^{\prime}\right)\right) \\
& =\int_{X} \varrho_{P, k} d \mu_{P, Q}\left(\cdot \times \Omega^{\prime}\right) .
\end{aligned}
$$

Hence we obtain

$$
\begin{aligned}
\tau\left(A_{P}^{1 / 2} A_{Q} A_{P}^{1 / 2}\right) & =\int_{X \times Y} \varrho_{P, k} \varrho_{Q, k} d \mu_{P Q} \leq \int_{X \times Y} \varrho_{P} \varrho_{Q} d \mu_{P Q} \\
& =\int_{X \times Y} \frac{d \nu_{P}^{\rho}}{d \mu_{P}} \frac{d \nu_{Q}^{\rho}}{d \mu_{Q}} d \mu_{P Q} \\
& \leq \int_{X \times Y} d \nu_{P}^{\rho} \otimes d \nu_{Q}^{\rho} \\
& =\nu_{P}^{\rho}(X) \nu_{Q}^{\rho}(Y)=\tau(\rho)^{2}=1
\end{aligned}
$$


Then by Lemma 3.2, it holds that

$$
\tau\left(e^{\ln A_{P}+\ln A_{Q}}\right) \leq \tau\left(A_{P}^{1 / 2} A_{Q} A_{P}^{1 / 2}\right) \leq 1,
$$

where

$$
A=\ln A_{P}+\ln A_{Q}
$$

is the unique self-adjoint operator associated to the closed quadratic form $q_{\ln A_{P}}+q_{\ln A_{Q}}$ on the closure $V$ of $D(A)=D\left(\ln _{-}^{1 / 2} A_{P}\right) \cap D\left(\ln _{-}^{1 / 2} A_{Q}\right)$; see [9] and [13, VIII.6]

We suppose now that $\rho$ writes $\rho=\sum_{i} p_{i} \Pi_{f_{i}}$ with orthonomal functions $f_{i}$, and satisfies

$$
\int_{X} \ln \varrho_{P} d \nu_{P}^{\rho}<\infty \text { and } \int_{Y} \ln -\varrho_{Q} d \nu_{Q}^{\rho}<\infty .
$$

Note that otherwise (11) is already satisfied. We show that $f_{i} \in D(A)$ for any $i$, and evaluate $\left\langle A f_{i}, f_{i}\right\rangle$. We can use a Jensen-type inequality for operator convex functions on POVM due to Choi, see [3, 12]. Namely, since $-\ln t$ is operator convex (see [1, Chap.V], it holds that

$$
-\ln A_{P} \leq-\int_{X} \ln \varrho_{P, k} d P,
$$

hence

$$
\begin{aligned}
-\left\langle\ln A_{P} f_{i}, f_{i}\right\rangle & \leq-\int_{X} \ln \varrho_{P, k} d\left\langle P f_{i}, f_{i}\right\rangle=-\int_{X} \ln \varrho_{P, k} d \nu_{P}^{\Pi_{f}} \\
& \leq \int_{X} \ln _{-} \varrho_{P} d \nu_{P}^{\rho}<\infty
\end{aligned}
$$

by assumption on $\rho$. One gets a similar inequality for $-\left\langle\ln A_{Q} f_{i}, f_{i}\right\rangle$. We can then apply Lemma 3.1 to $-A$ on $V$ with $\Pi_{V} \rho \Pi_{V}=\rho$. Summing (7) and using (6) yields

$$
-\int_{X} \ln \varrho_{P, k} d \nu_{P}^{\rho}-\int_{Y} \ln \varrho_{Q, k} d \nu_{Q}^{\rho} \geq \tau(-A \rho) \geq S(\rho),
$$

and gives Theorem 1.1 by monotone convergence when $k \rightarrow+\infty$.

Equality case. We discuss here the equality case in Theorem 1.1. It holds iff (5), (7), (8) become sharp for $k \rightarrow \infty$. Equality in (5) means that the Liouville measure is a product, i.e. the POVM $P$ and $Q$ are independent. By [12], (7) are all equalities iff $P$ and $Q$ are projection valued measures on the support of $\nu_{P}^{\rho}$ and $\nu_{Q}^{\rho}$. Then equality holds in (\&) iff it holds:

- in Golden-Thompson inequality, which is achieved when $P$ and $Q$ commute,

- and in Gibbs formula, meaning that $A_{P} A_{Q} \rightarrow \rho$, i.e. $\rho$ is a product state with respect to $P, Q$.

Altogether these conditions are very restrictive. Examples are given by a space $H$ that splits into $H_{1} \otimes H_{2}$ with given basis $\left(e_{i}\right),\left(f_{j}\right)$. There $P(i)=\Pi_{e_{i}} \otimes 1$ and $Q(j)=1 \otimes \Pi_{f_{j}}$, and product states $\rho=\rho_{1} \otimes \rho_{2}$ give equalities.

Note however that an approximate equality in (11) may be achieved in other cases. This happens for instance for the Fourier transform discussed in $\$ 2.2$. Here the position-momentum maps are independent projection valued measures, hence (5) and (77) are equalities. Although $P$ and $Q$ don't commute here, Theorem 1.1 becomes sharp on states that spread at large scale on the $(x, \xi)$ phase space like $\rho_{t}=e^{-t\left(\Delta+\|x\|^{2}\right)} / \tau\left(e^{-t\left(\Delta+\|x\|^{2}\right)}\right)$ when $t \searrow 0$; see [14. 


\section{Applications on homogeneous spaces}

4.1. Invariant operators and spatial/spectral independence. We turn back to examples and describe a setting on homogeneous spaces leading to independent spatial and spectral projections valued measures.

Let $H$ be a closed group in a locally compact group $G$ and let $X=G / H$ be the corresponding homogeneous space. We assume moreover that $X$ admits a $G$-invariant Borel measure $\mu$. This is equivalent to the equality of modular functions $\delta_{H}=\delta_{G}$ on $H$, and $\mu$ is unique up to constants, see [8, 10].

Let $V$ be a separable Hilbert space and consider $\mathcal{H}=L^{2}(X, V, \mu)$. We say that an isometry $U$ of $\mathcal{H}$ is a gauge transform if it acts fiberwise over $X$, i.e. $(U f)(x)=U_{x}(f(x))$ for some isometries $U_{x}: V \rightarrow V$.

Definition 4.1. An operator $A$ on $\mathcal{H}$ will be called translation gauge-invariant, if it is conjugated by a gauge transform $U_{0}$ to an operator $A_{0}=U_{0}^{-1} A U_{0}$ whose translates $A_{0}^{g}=g^{-1} A_{0} g$ stay conjugated to $A_{0}$ up to gauge transforms, i.e. $A_{0}^{g}=U(g)^{-1} A_{0} U(g)$.

This class contains the translation invariant operators on $X$, as differential operators with constant coefficients when $G$ is a Lie group. It contains also operators conjugated to translation invariant operators by gauge transform; for instance $A=i \frac{d}{d x}+x=e^{-i x^{2} / 2} i \frac{d}{d x} e^{i x^{2} / 2}$ is not translation-invariant, yet translation gauge-invariant on $L^{2}(\mathbb{R})$. Another classical example is given by the Hamiltonian of a constant magnetic field $B \geq 0$ in $\mathbb{R}^{2}$

$$
H_{B}=\left(-i \frac{\partial}{\partial x}+\frac{B y}{2}\right)^{2}+\left(-i \frac{\partial}{\partial y}-\frac{B x}{2}\right)^{2}=(-i \nabla+A)^{2} .
$$

A general gauge transform acts on $H_{B}$ as $e^{-i f} H_{B} e^{i f}=(-i \nabla+A+d f)^{2}$, and fixing $f(x, y)=$ $\frac{B}{2}\left(y_{0} x-x_{0} y\right)$ actually translates $H_{B}$ on $\mathbb{R}^{2}$ by $\left(x_{0}, y_{0}\right)$

Our interest for gauge invariant self-adjoint operators $A$ here comes from the independence of the projection valued measure on $H$ coming from $X$; namely $P(\Omega)=\chi_{\Omega} \times$, and the spectral resolution of $A$ on $Y=\mathbb{R}: Q(I)=\Pi_{A}(I)$.

Proposition 4.2. Let $A$ be a translation gauge-invariant self-adjoint operator on $L^{2}(X, V, \mu)$. Then there exists a measure $\mu_{A}$ on $\mathbb{R}$ such that the Liouville measure writes

$$
\mu_{P Q}=\mu \otimes \mu_{A}
$$

We shall call $\mu_{A}$ the spectral measure of $A$ in the sequel (not to be confused with the projection valued measure $Q=\Pi_{A}$ ).

Proof. We first observe that conjugating any $A$ by a gauge transform preserves $\mu_{P Q}$. Indeed if $A^{U}=U^{-1} A U$ then $\Pi_{A^{U}}(I)=U^{-1} \Pi_{A}(I) U$ and

$$
\begin{aligned}
\mu_{P Q^{U}}(\Omega \times I) & =\tau\left(\chi_{\Omega} \Pi_{A^{U}}(I)\right)=\tau\left(U \chi_{\Omega} U^{-1} \Pi_{A}(I)\right) \\
& =\tau\left(\chi_{\Omega} \Pi_{A}(I)\right)=\mu_{P Q}(\Omega \times I) .
\end{aligned}
$$


Then if $g^{-1} A g=A^{U(g)}$, one has

$$
\begin{aligned}
\mu_{P Q}(g . \Omega \times I) & =\tau\left(\chi_{g \Omega} \Pi_{A}(I)\right)=\tau\left(g \chi_{\Omega} g^{-1} \Pi_{A}(I)\right) \\
& =\tau\left(\chi_{\Omega} g^{-1} \Pi_{A}(I) g\right)=\tau\left(\chi_{\Omega} \Pi_{A^{U}}(I)\right) \\
& =\mu_{P Q}(\Omega \times I) .
\end{aligned}
$$

Hence, given $I, \Omega \mapsto \mu_{P Q}(\Omega \times I)$ is an invariant measure on $X$ thus proportional to $\mu$ by uniqueness, i.e. $\mu_{P Q}(\Omega \times I)=\mu(\Omega) \mu_{A}(I)$ as needed.

More concretely, $\mu_{A}(I)$ expresses using the Schwarz kernel $K_{\Pi_{A}(I)}$ of $\Pi_{A}(I)$. Indeed

$$
\begin{aligned}
\mu_{P Q}(\Omega \times I) & =\tau_{\mathcal{H}}\left(\chi_{\Omega} \Pi_{A}(I)\right)=\left\|\chi_{\Omega} \Pi_{A}(I)\right\|_{H S(\mathcal{H})}^{2} \\
& =\int_{\Omega} \int_{X}\left\|K_{\Pi_{A}(I)}(x, y)\right\|_{H S(V)}^{2} d \mu(y) d \mu(x),
\end{aligned}
$$

so that one has for $\mu$-almost every $x$

$$
\mu_{A}(I)=\int_{X}\left\|K_{\Pi_{A}(I)}(x, y)\right\|_{H S(V)}^{2} d \mu(y) .
$$

This last formula, together with Plancherel formula on $X$, helps in computing examples.

4.2. Compact case. We first consider the case of homogeneous spaces $X=G / H$ for compact groups $G$. Then a scalar invariant self-adjoint operator $A$ on $X$ induces a spectral splitting

$$
L^{2}(X)=\bigoplus_{\operatorname{Sp} A} E_{\lambda}
$$

The spectral measure $\mu_{A}$ is supported on $\operatorname{Sp} A$ and

$$
\begin{aligned}
\mu_{P Q}\left(X \times \Pi_{\lambda}\right) & =\tau\left(\Pi_{\lambda}\right)=\operatorname{dim} E_{\lambda} \\
& =\mu(X) \mu_{A}(\lambda)
\end{aligned}
$$

hence $\mu_{A}(\lambda)=\operatorname{dim} E_{\lambda} / \mu(X)$. Therefore (11) writes here

$$
-\int_{X} \varrho(x) \ln \varrho(x) d \mu(x)-\sum_{\operatorname{Sp} A} \ln \left(\frac{\tau\left(\rho \Pi_{\lambda}\right)}{\operatorname{dim} E_{\lambda}}\right) \tau\left(\rho \Pi_{\lambda}\right) \geq S(\rho),
$$

if $\mu$ is normalized such that $\mu(X)=1$.

A first remark here is that the spectral entropy sum, say $S_{A}(\rho)$, actually does not depend on the values in $\operatorname{Sp} A$. It stays the same for any other operator $\varphi(A)$ with $\varphi$ injective on $\operatorname{Sp} A$. This sum only depends on the repartition on the state in the splitting (12).

This stays true in general for the change of self-adjoint operator $A$ into $\varphi(A)$ on any space $X$. Indeed one has $\Pi_{\varphi(A)}(I)=\Pi_{A}\left(\varphi^{-1}(I)\right)$ and the induced projection valued measure becomes $Q_{\varphi(A)}=Q_{A} \circ \varphi^{-1}$. One sees easily (or by Proposition 4.3 below) that this amounts in a change of variables $\lambda \mapsto \varphi^{-1}(\lambda)$ in the spectral entropy integrals over $Y=\mathbb{R}$ in (1), and thus $S_{A}(\rho)=S_{\varphi(A)}(\rho)$. Hence this notion does not depend on the actual values of energy levels, not even their order, but deals with the repartition of the state in the direct integral splitting of $H=\int^{\oplus} d E_{\lambda}$. 
We also observe that (13) is sharp on purely spectral states such that $\rho=\varphi(A)$ with $\tau(\rho)=1$. Indeed these translation invariant states have constant density $\varrho(x)=1$ since $\mu(X)=1=\tau(\rho)$. Thus the spatial entropy term vanishes in (13), while

$$
S_{A}(\rho)=S(\rho)=-\sum_{\operatorname{Sp} A}(\ln \varphi(\lambda)) \varphi(\lambda) \operatorname{dim} E_{\lambda} .
$$

Finally, we note that $S_{Q}(\rho)$ decreases with the refinement of the splitting of $L^{2}(X)$ and is ultimately bounded from below by the finest possible one coming from the decomposition of the unitary representation $\pi$ of $G$ in $L^{2}(X)$ into finite dimensional irreducible representations:

$$
L^{2}(X)=\bigoplus_{\sigma \subset \pi} H_{\sigma}
$$

This comes from the splitting of each invariant space $E_{\lambda}$ into such sums of $H_{\sigma}$ (explaining the discreteness of $\operatorname{Sp} A$ ), and the following general monotony property.

Proposition 4.3. Let $P$ be a POVM from $(X, \mu)$ to $H$. Suppose that $P^{\prime}$ is a POVM on $\left(X^{\prime}, \mu^{\prime}\right)$ induced from $P$ by a measurable map $\varphi: X \rightarrow X^{\prime}$, i.e. $P^{\prime}=P\left(\varphi^{-1}\right)$ and $\mu^{\prime}=\mu\left(\varphi^{-1}\right)$. Suppose moreover that $X^{\prime}$ is $\sigma$-finite. Then

$$
S_{P^{\prime}}(\rho)=-\int_{X^{\prime}} \ln \left(\frac{d \nu_{P^{\prime}}^{\rho}}{d \mu^{\prime}}\right) d \nu_{P^{\prime}}^{\rho} \geq S_{P}(\rho)=-\int_{X} \ln \left(\frac{d \nu_{P}^{\rho}}{d \mu}\right) d \nu_{P}^{\rho},
$$

provided these integrals are defined.

Indeed, Jensen inequality applied to the conditional expectation $E\left(\frac{d \nu_{P}^{\rho}}{d \mu} \mid \varphi\right)=\frac{d \nu_{P^{\prime}}^{\rho}}{d \mu^{\prime}}$ gives

$$
E\left(-\frac{d \nu_{P}^{\rho}}{d \mu} \ln \left(\frac{d \nu_{P}^{\rho}}{d \mu}\right) \mid \varphi\right) \leq-\frac{d \nu_{P^{\prime}}^{\rho}}{d \mu^{\prime}} \ln \left(\frac{d \nu_{P^{\prime}}^{\rho}}{d \mu^{\prime}}\right)
$$

yielding (15) by integration.

Eventually we illustrate this discussion on the sphere $S^{n-1}=\mathrm{SO}(n) / \mathrm{SO}(n-1)$ and the Laplacian $\Delta_{S}$. Here $\operatorname{Sp}\left(\Delta_{S}\right)=\{d(d+n-2) \mid d \geq 0\}$ and $E_{d}(\Delta)$ consists in the harmonic polynomials of degree $d$, with $\operatorname{dim} E_{d}=\left(\begin{array}{c}d+n-1 \\ n-1\end{array}\right)-\left(\begin{array}{c}d+n-3 \\ n-1\end{array}\right)$, see e.g. [8, 16]. Moreover the representation of $\mathrm{SO}(n)$ on $E_{d}$ is irreducible, so that the spectral and Weyl decompositions (12) and (14) coincide here. That means that the measurement of $\rho$ by $\Delta_{S}$ (its energy distribution), actually provides the best (lowest) spectral entropic term (among invariant operators) in (13).

4.3. Non-compact examples. We compute the spectral measure of some operators in noncompact situations. Let $A$ be a self-adjoint differential operator with constant coefficients on $X=\mathbb{R}^{n}$ and $\sigma_{A}$ its polynomial symbol. The Fourier transform of the spectral projection $\Pi_{A}(I)$ is the multiplication by $\chi_{\sigma_{A}^{-1}(I)}$ on $\mathbb{R}_{\xi}^{n}$. Then by (11) and Plancherel formula the spectral measure of $A$ is

$$
\mu_{A}(I)=\left\|\chi_{\sigma^{-1}(I)}\right\|_{L_{\xi}^{2}}^{2}=\operatorname{vol}\left(\sigma_{A}^{-1}(I)\right) .
$$

Using the coarea formula, its density with respect to Lebesgue measure on $\mathbb{R}$ is

$$
\frac{d \mu_{A}}{d \lambda}=\int_{\sigma_{A}^{-1}(\lambda)} \frac{d H_{\sigma}}{|\nabla \sigma|}
$$


for the hypersurface measure $H_{\sigma}$ on $\sigma^{-1}(\lambda)$. Similarly the measure of a state $\rho=\sum_{i} p_{i} \Pi_{f_{i}}$ in $L^{2}\left(\mathbb{R}^{n}\right)$ relatively to the spectral resolution of $A$ reads

$$
\nu_{A}^{\rho}(I)=\tau\left(\rho \Pi_{A}(I)\right)=\int_{\sigma_{A}^{-1}(I)} \widehat{\varrho}(\xi) d \xi
$$

where $\widehat{\varrho}(\xi)=\sum_{i} p_{i}\left|\widehat{f}_{i}(\xi)\right|^{2}$ as in $\$ 2.2$. Hence

$$
\frac{d \nu_{A}^{\rho}}{d \mu_{A}}(\lambda)=\int_{\sigma_{A}^{-1}(\lambda)} \widehat{\varrho}(\xi) \frac{d H_{\sigma}}{|\nabla \sigma|} / \int_{\sigma_{A}^{-1}(\lambda)} \frac{d H_{\sigma}}{|\nabla \sigma|},
$$

expliciting the spectral entropy term in (四):

$$
S_{A}(\rho)=-\int_{\mathbb{R}} \ln \left(\frac{d \nu_{A}^{\rho}}{d \mu_{A}}\right) d \nu_{A}^{\rho} .
$$

For instance in the case of the Laplacian $\Delta$. Then $\sigma_{\Delta}(\xi)=\|2 \pi \xi\|^{2}$ with our convention in $\$ 2.2$, and the spectral measure is $\nu_{\Delta}([0, \lambda])=\operatorname{vol}(B(\sqrt{\lambda} / 2 \pi))$. Then $H_{\sigma}$ is the usual measure $m$ on the spheres $S_{R}$, and one finds that

$$
S_{\Delta}(\rho)=-\int_{0}^{\infty} \ln \left(\int_{S_{R}} \widehat{\varrho}(\xi) \frac{d m(\xi)}{m\left(S_{R}\right)}\right)\left(\int_{S_{R}} \widehat{\varrho}(\xi) d m(\xi)\right) d R
$$

We note that for any invariant $A$, the map $\Pi_{A}$ is actually induced (up to Fourier transform) from the momentum map $Q(\Omega)=\chi_{\Omega} \times$ on $\mathbb{R}_{\xi}^{n}$ by the symbol $\sigma_{A}: \mathbb{R}_{\xi}^{n} \rightarrow \mathbb{R}$; i.e. $\Pi_{A}=Q\left(\sigma_{A}^{-1}\right)$ and $\mu_{A}=\mu_{Q}\left(\sigma_{A}^{-1}\right)$ by (16). Hence Proposition 4.3 applies and gives the lower bound

$$
S_{A}(\rho) \geq S_{Q}(\rho)=-\int_{\mathbb{R}^{n}} \widehat{\varrho}(\xi) \ln \widehat{\varrho}(\xi) d \xi,
$$

arising in the Fourier uncertainty principle (3). Yet, contrarily to the Laplacian on $S^{n-1}$, one has in general $S_{A}(\rho)>S_{Q}(\rho)$, as due to Jensen inequality. Indeed the knowledge of $S_{A}(\rho)$ requires less information on $\rho$ than $S_{Q}(\rho)$, since $\nu_{A}^{\rho}$ only depends on the mean values of $\varrho$ on the level sets of $\sigma_{A}$.

We mention that the spectral measure $\mu_{A}$ can (in principle) be computed on other noncompact homogeneous spaces $X=G / H$, as long an explicit Plancherel formula is available. This is indeed the case for symmetric spaces $G / K$ with $G$ connected semi-simple Lie group with finite center and $K$ its maximal compact subgroup; see e.g. [8]. For instance, computations of the spectral measure of the Laplacian on symmetric spaces may be found in [16].

We close this series with the example (9) of the Hamiltonian $H_{B}$ in $L^{2}\left(\mathbb{R}^{2}\right)$. It is translation gauge-invariant and Proposition 4.1 applies. Its spectrum is discrete and consists in the (Landau levels) $\lambda_{n}=(2 n+1) B, n \in \mathbb{N}$, with each eigenspace $E_{n}$ of constant density $\mu_{H_{B}}\left(\lambda_{n}\right)=\frac{B}{2 \pi}$; see e.g. [6]. Hence (11) reads here

$$
-\int_{\mathbb{R}^{2}} \varrho(x) \ln \varrho(x) d x-\sum_{n \geq 0} \tau\left(\rho \Pi_{n}\right) \ln \tau\left(\rho \Pi_{n}\right) \geq S(\rho)-\ln (B / 2 \pi) .
$$

One sees that the uncertainty constrain relaxes for a given state when $B$ increases. Indeed the state may concentrate on fewer Landau levels, whose density increases with $B$. Note also 
that the spectral entropy term in the left side is always positive here and vanishes iff $\rho$ is contained in a single level, in which case $-\int_{\mathbb{R}^{2}} \varrho(x) \ln \varrho(x) d x \geq S(\rho)-\ln (B / 2 \pi)$.

4.4. Log-Sobolev inequalities. As emphasized above, the spectral entropy associated to an operator does not depend on the actual values of energy levels, but only on the induced spectral splitting. It turns out however that one can bound it using a single estimation of the mean energy of the state $\mathcal{E}_{A}(\rho)=\tau(A \rho)$; yielding a log-Sobolev (entropy-energy) inequality.

Consider again a self-adjoint translation gauge-invariant operator $A$ on an homogeneous space $X=G / H$. For $t \geq 0$, let

$$
L_{A}(t)=\int_{\mathbb{R}} e^{-t \lambda} d \mu_{A}(\lambda)
$$

denotes the Laplace transform of the spectral measure $\mu_{A}$. This is actually also the constant ratio $\tau\left(e^{-t A} \chi_{\Omega}\right) / \mu(\Omega)$, or heat decay of $e^{-t A}$. The following Gibbs inequality holds.

Proposition 4.4. Suppose that the state $\rho$ satisfies $\mathcal{E}_{A}^{+}(\rho)=\tau(\max (A, 0) \rho)<\infty$ and that $L(t)<\infty$. Then

$$
\int_{\mathbb{R}} \ln ^{-}\left(\frac{d \nu_{A}^{\rho}}{d \mu_{A}}\right) d \mu_{A}(\rho)<\infty
$$

and it holds that

$$
S_{A}(\rho) \leq t \mathcal{E}_{A}(\rho)+\ln L_{A}(t) .
$$

Proof. From $\mathcal{E}_{A}(\rho)=\tau(\rho A)=\int_{\mathbb{R}} \lambda \tau\left(\rho d \Pi_{A}\right)=\int_{\mathbb{R}} \lambda d \nu_{A}^{\rho}$, one has

$$
\begin{aligned}
-\int_{\mathbb{R}}\left(\frac{d \nu_{A}^{\rho}}{e^{-t \lambda} d \mu_{A}}\right) \ln \left(\frac{d \nu_{A}^{\rho}}{e^{-t \lambda} d \mu_{A}}\right) e^{-t \lambda} d \mu_{A} & =S_{A}(\rho)-t \int_{\mathbb{R}} \lambda d \nu_{A}^{\rho} \\
& =S_{A}(\rho)-t \mathcal{E}_{A}(\rho) \\
& \leq-\left(\int_{\mathbb{R}} d \nu_{A}^{\rho}\right) \ln \left(\int_{\mathbb{R}} \frac{d \nu_{A}^{\rho}}{L(t)}\right)=\ln L(t),
\end{aligned}
$$

by Jensen inequality and $\int_{\mathbb{R}} d \nu_{A}^{\rho}=\nu_{A}^{\rho}(\mathbb{R})=\tau(\rho)=1$. Note that $\ln ^{-}\left(\frac{d \nu_{A}^{\rho}}{d \mu_{A}}\right)-t \lambda^{+} \leq$ $(-\ln )^{+}\left(\frac{d \nu_{A}^{\rho}}{e^{-t \lambda} d \mu_{A}}\right)$ whose corresponding integral is finite by Jensen if $L_{A}(t)<\infty$. This ensures that $S_{A}(\rho)$ exists.

Remark 4.5. Note moreover that equality holds iff $d \nu_{A}^{\rho}=C e^{-t \lambda} d \mu_{A}=e^{-t \lambda} d \mu_{A} / L_{A}(t)$, i.e. when the spectral distribution of the state is a Gibbs measure, i.e. with exponential law with respect to energy. Such states always exist in the homogeneous case: take for instance

$$
\rho_{t}=e^{-\frac{t}{2} A} \chi_{\Omega} e^{-\frac{t}{2} A} / L_{A}(t) \mu(\Omega) .
$$

A corollary is the following log-Sobolev inequality, in a parametric form.

Corollary 4.6. Under the previous assumptions, it holds that

$$
-\int_{X} \varrho(x) \ln \varrho(x) d \mu(x)+t \mathcal{E}_{A}(\rho)+\ln L_{A}(t) \geq S(\rho) .
$$


When $\rho$ is a pure state $\Pi_{f}$ and $A$ is the Laplacian (or $e^{-t A}$ is Markovian) this is a well known inequality, see [⿶]. It extends here for mixed states on a large class of operators: translation gauge-invariant ones on homogeneous manifolds.

One can also optimize (21) in $t$. Notice that $\ln L_{A}(t)$ is a convex function, since $L_{A}(t)$ is the integral of log-convex functions. We consider then the Legendre transform (Young conjugate) of $-\ln L_{A}(t)$, namely

$$
\left(\ln L_{A}\right)^{*}(\lambda)=\inf _{t \geq 0}\left(t \lambda+\ln L_{A}(t)\right) .
$$

Then (21) reads

$$
-\int_{X} \varrho(x) \ln \varrho(x) d \mu(x)+\left(\ln L_{A}\right)^{*}\left(\mathcal{E}_{A}(\rho)\right) \geq S(\rho) .
$$

Note that by Remark 4.5, (22) is also equivalent to the previous purely entropic inequality

$$
-\int_{X} \varrho(x) \ln \varrho(x) d \mu(x)+S_{A}(\rho) \geq S(\rho)
$$

on states at statistical equilibrium with respect to the energy $A$, i.e. with spectral measure $d \nu_{A}^{\rho}=C e^{-t \lambda} d \mu_{A}$ for some $t$, but is weaker on more general states. Moreover, following the discussion in $\$ 4.2$, the inequality (22) is sharp (on mixed states) on homogeneous spaces $X=G / H$ with $G$ compact, if $e^{-t A}$ is trace class for some $t$.

Examples. In the case of the Laplacian on $\mathbb{R}^{n}$, one has $L_{\Delta}(t)=(4 \pi t)^{-n / 2}$; a well known heat-decay (that also follows from (19) with $\nu_{\Delta}([0, \lambda])$ given in $\left.\$ 4.3\right)$. Then (22) writes

$$
-\int_{X} \varrho(x) \ln \varrho(x) d \mu(x)+\frac{n}{2} \ln \left(\frac{e \mathcal{E}_{\Delta}(\rho)}{2 \pi n}\right) \geq S(\rho) .
$$

This inequality is due to Dolbeault-Felmer-Loss-Paturel in [5] and asymptotically sharp on normalized harmonic oscillator heat $\rho_{t}=e^{-t\left(\Delta+\|x\|^{2}\right)} / \tau\left(e^{-t\left(\Delta+\|x\|^{2}\right)}\right)$ for $t \searrow 0$.

We can compare this on $\mathbb{R}^{2}$ with the Hamiltonian $H_{B}$ in $(9)$. From discussion in $\$ 4.3$, one has here

$$
L_{B}(t)=\sum_{n \geq 0} \frac{B}{2 \pi} e^{-(2 n+1) B t}=\frac{B}{4 \pi \sinh (B t)} .
$$

Straightforward calculations then yields

$$
\left(\ln L_{B}\right)^{*}\left(\mathcal{E}_{H_{B}}(\rho)\right)=\ln \left(\frac{\mathcal{E}_{H_{B}}(\rho)+B}{4 \pi}\right)+n(\rho) \ln \left(1+\frac{1}{n(\rho)}\right),
$$

where

$$
n(\rho)=\sum_{n} n \tau\left(\Pi_{n} \rho\right)
$$

is the mean Landau level of $\rho$ (with respect to the ground state). Note that

$$
\mathcal{E}_{H_{B}}(\rho)=(2 n(\rho)+1) B .
$$

When $B \rightarrow 0$, one has $H_{B} \rightarrow \Delta$ and $n(\rho) \rightarrow+\infty$ for a given state. Then

$$
\left(\ln L_{B}\right)^{*}\left(\mathcal{E}_{H_{B}}(\rho)\right) \rightarrow \ln \left(\frac{e \mathcal{E}_{\Delta}(\rho)}{4 \pi}\right)
$$

and one recovers $(23)$ on $\mathbb{R}^{2}$ from $(22)$. 
Further comments. The log-Sobolev inequality (22) obtained here may be compared to an other similar result proved in [14]. There Corollary 1.6 states that

$$
-\int_{X} \varrho(x) \ln \varrho(x) d \mu(x)+\left(\ln F_{A}\right)^{c}\left(\mathcal{E}_{A}(\rho)\right) \geq-3-\ln \|\rho\|_{L^{2} \rightarrow L^{2}}
$$

where

$$
F_{A}(\lambda)=\sup _{\Omega}\left(\frac{\tau\left(\chi_{\Omega} \Pi_{A}(]-\infty, \lambda[)\right.}{\mu(\Omega)}\right)
$$

and $\left(\ln F_{A}\right)^{c}$ denotes the concave hull of $\ln F_{A}$. This statement holds on general $\sigma$-finite spaces without invariance and homogeneity assumptions.

In an homogenous situation, one has $F_{A}(\lambda)=\mu_{A}(]-\infty, \lambda[)$. It turns out that

$$
\left(\ln L_{A}\right)^{*} \geq\left(\ln F_{A}\right)^{c}
$$

so that the left side of (22) is larger than the one in (25). Indeed,

$$
\begin{aligned}
L_{A}(t) & =\int_{\mathbb{R}} e^{-t u} d \mu_{A}(u) \\
& \geq \int_{-\infty}^{\lambda} e^{-t \lambda} d \mu_{A}(u)=e^{-t \lambda} F_{A}(\lambda)
\end{aligned}
$$

and thus $\ln F_{A}(\lambda) \leq t \lambda+\ln L_{A}(t)$, giving (26) by concavity of $\left(\ln L_{A}\right)^{*}$. On the other hand the right side of (25) is smaller than in (22) since on unit trace states

$$
-\ln \|\rho\|_{L^{2} \rightarrow L^{2}} \leq S(\rho)=-\tau(\rho \ln \rho),
$$

with equality on uniformly distributed states, i.e. normalized projections on finite dimensional subspaces of $H$.

In conclusion the two log-Sobolev inequalities we discuss here are not equivalent, even on homogeneous spaces; see also [14, §4.2] for a more precise comparison in the case of the Laplacian on $\mathbb{R}^{n}$. Yet, we have seen that the version developped here is sharp in some classical cases, including translation gauge-invariant operators on compact homogeneous spaces. Moreover it comes from the stronger entropic uncertainty principle stated in Theorem 1.1.

Acknowledgments. The author is grateful to Rupert L. Frank and Elliott H. Lieb for showing him their earlier proof of the Fourier uncertainty inequality (3).

\section{REFERENCES}

[1] R. Bhatia. Matrix analysis, volume 169 of Graduate Texts in Mathematics. Springer-Verlag, New York, 1997.

[2] E. Carlen. Trace inequalities and quantum entropy: an introductory course. In Entropy and the quantum, volume 529 of Contemp. Math., pages 73-140. Amer. Math. Soc., Providence, RI, 2010.

[3] M. D. Choi. A Schwarz inequality for positive linear maps on $C^{*}$-algebras. Illinois J. Math., 18:565-574, 1974.

[4] E. B. Davies. Heat kernels and spectral theory, volume 92 of Cambridge Tracts in Mathematics. Cambridge University Press, Cambridge, 1989.

[5] J. Dolbeault, P. Felmer, M. Loss, and E. Paturel. Lieb-Thirring type inequalities and Gagliardo-Nirenberg inequalities for systems. J. Funct. Anal., 238(1):193-220, 2006.

[6] L. Erdős, M. Loss, and V. Vougalter. Diamagnetic behavior of sums of Dirichlet eigenvalues. Ann. Inst. Fourier (Grenoble), 50(3):891-907, 2000. 
[7] R. L. Frank and E. H. Lieb. Entropy and the uncertainty principle. Preprint arXiv http://fr.arxiv. org/abs/1109.1209.

[8] S. Helgason. Groups and geometric analysis, volume 113 of Pure and Applied Mathematics. Academic Press Inc., Orlando, FL, 1984. Integral geometry, invariant differential operators, and spherical functions.

[9] T. Kato. Trotter's product formula for an arbitrary pair of self-adjoint contraction semigroups. In Topics in functional analysis (essays dedicated to M. G. Krě̆n on the occasion of his 70th birthday), volume 3 of Adv. in Math. Suppl. Stud., pages 185-195. Academic Press, New York, 1978.

[10] L. Nachbin. The Haar integral. Robert E. Krieger Publishing Co., Huntington, N.Y., 1976. Translated from the Portuguese by Lulu Bechtolsheim, Reprint of the 1965 edition.

[11] M. A. Nielsen and I. L. Chuang. Quantum computation and quantum information. Cambridge University Press, Cambridge, 2000.

[12] D. Petz. On the equality in Jensen's inequality for operator convex functions. Integral Equations Operator Theory, 9(5):744-747, 1986.

[13] M. Reed and B. Simon. Methods of modern mathematical physics. I. Academic Press Inc. [Harcourt Brace Jovanovich Publishers], New York, second edition, 1980. Functional analysis.

[14] M. Rumin. Balanced distribution-energy inequalities and related entropy bounds. Preprint 2010. To appear in Duke Math Journal.

[15] B. Simon. Trace ideals and their applications, volume 120 of Mathematical Surveys and Monographs. American Mathematical Society, Providence, RI, second edition, 2005.

[16] R. S. Strichartz. Estimates for sums of eigenvalues for domains in homogeneous spaces. J. Funct. Anal., 137(1):152-190, 1996

[17] Wikipedia. Mutually unbiased bases. http://en.wikipedia.org/wiki/Mutually_unbiased_bases.

[18] Wikipedia. POVM. http://en.wikipedia.org/wiki/Positive_operator-valued_measure.

Laboratoire de Mathématiques d'Orsay, UMR 8628, CNRS et Université Paris-Sud, 91405 ORsAy, FrANCE

E-mail address: michel.rumin@math.u-psud.fr 